University of Wollongong

Research Online

Faculty of Social Sciences - Papers (Archive) Faculty of Arts, Social Sciences \& Humanities

$1-1-2016$

The Montreal Cognitive Assessment (MoCA) is sensitive to head injury and cognitive impairment in a residential alcohol and other drug therapeutic community

Ely Marceau

University of Wollongong, emm993@uowmail.edu.au

Jo Lunn

We Help Ourselves

Jamie Berry

Advanced Neuropsychological Treatment Services

Peter Kelly

University of Wollongong, pkelly@uow.edu.au

Nadia Solowij

University of Wollongong, nadia@uow.edu.au

Follow this and additional works at: https://ro.uow.edu.au/sspapers

Part of the Education Commons, and the Social and Behavioral Sciences Commons

Research Online is the open access institutional repository for the University of Wollongong. For further information contact the UOW Library: research-pubs@uow.edu.au 


\title{
The Montreal Cognitive Assessment (MoCA) is sensitive to head injury and cognitive impairment in a residential alcohol and other drug therapeutic community
}

\begin{abstract}
Introduction: Retaining clients in residential alcohol and other drug (AOD) treatment is difficult and cognitive impairment has been identified as a significant predictor of treatment dropout. The application of extensive screening for cognitive impairment is cost-prohibitive for most AOD treatment services. The current study aimed to explore cognitive functioning and impairment-associated factors in a typical sample of residential AOD clients using a free brief screening tool that could be utilised by front-line AOD services. Methods: Residents of an AOD therapeutic community $(n=128)$ and a non-substance using control group $(n=37)$ were administered a brief cognitive screening measure, the Montreal Cognitive Assessment (MoCA). MoCA total and domain scores were compared between these groups and within the AOD group examined in association with primary substance of misuse, severity of dependence, gender, psychological distress, and history of head injury. Results: Almost half (43.8\%) of the AOD sample were identified as cognitively impaired, compared to $16.2 \%$ of the control group. Furthermore, $67.2 \%$ of the AOD sample had sustained head injuries and $50 \%$ of the sample required hospitalization for head injury. History of head injury was a significant determinant of cognitive impairment, and associated with greater levels of psychological distress. Conclusions: There are high rates of inter-related cognitive impairment, head injuries, and psychological distress among clients in residential AOD treatment. Routine screening of clients at intake for cognitive impairment by means of a brief screening measure such as the MoCA, in combination with the assessment of history of head injuries and comorbid psychological disorders, could inform treatment modifications or adjunct interventions to increase retention and improve long-term outcomes.
\end{abstract}

\section{Keywords}

moca, assessment, cognitive, montreal, sensitive, head, injury, impairment, residential, therapeutic, alcohol, community, other, drug

Disciplines

Education | Social and Behavioral Sciences

\section{Publication Details}

Marceau, E. M., Lunn, J., Berry, J., Kelly, P. J. \& Solowij, N. (2016). The Montreal Cognitive Assessment (MoCA) is sensitive to head injury and cognitive impairment in a residential alcohol and other drug therapeutic community. Journal of Substance Abuse Treatment, 66 30-36. 
The Montreal Cognitive Assessment (MoCA) is sensitive to head injury and cognitive impairment in a residential alcohol and other drug therapeutic community

Ely M. Marceau ${ }^{\mathrm{a}}$, Jo Lunn ${ }^{\mathrm{b}}$, Jamie Berry ${ }^{\mathrm{c}}$, Peter J. Kelly ${ }^{\mathrm{a}}$, and Nadia Solowij ${ }^{\mathrm{a} *}$

${ }^{\text {a }}$ School of Psychology, Centre for Health Initiatives, and Illawarra Health and Medical

Research Institute, University of Wollongong, Northfields Ave Wollongong NSW 2522

Australia

${ }^{\mathrm{b}}$ We Help Ourselves (WHOs), Building 128, Church St Lilyfield NSW 2040 Australia

c Advanced Neuropsychological Treatment Services, PO Box 4070 Strathfield South NSW

2136 Australia

* Corresponding Author at:

School of Psychology, University of Wollongong

Northfields Ave Wollongong NSW 2522 Australia

Tel: +61242213732, Fax: +61242214163, E-mail: nadia@uow.edu.au 


\begin{abstract}
Introduction

Retaining clients in residential alcohol and other drug (AOD) treatment is difficult and cognitive impairment has been identified as a significant predictor of treatment dropout. The application of extensive screening for cognitive impairment is cost-prohibitive for most AOD treatment services. The current study aimed to explore cognitive functioning and impairmentassociated factors in a typical sample of residential AOD clients using a free brief screening tool that could be utilised by front-line AOD services.
\end{abstract}

\title{
Methods
}

Residents of an AOD therapeutic community $(n=128)$ and a non-substance using control group ( $n=37$ ) were administered a brief cognitive screening measure, the Montreal Cognitive Assessment (MoCA). MoCA total and domain scores were compared between these groups and within the AOD group examined in association with primary substance of misuse, severity of dependence, gender, psychological distress, and history of head injury.

Results

Almost half (43.8\%) of the AOD sample were identified as cognitively impaired, compared to $16.2 \%$ of the control group. Furthermore, $67.2 \%$ of the AOD sample had sustained head injuries and $50 \%$ of the sample required hospitalisation for head injury. History of head injury was a significant determinant of cognitive impairment, and associated with greater levels of psychological distress.

\section{Conclusions}

There are high rates of inter-related cognitive impairment, head injuries, and psychological distress among clients in residential AOD treatment. Routine screening of clients at intake for cognitive impairment by means of a brief screening measure such as the MoCA, in combination with the assessment of history of head injuries and comorbid psychological disorders, could inform treatment modifications or adjunct interventions to increase retention and improve long-term outcomes.

Key words: substance misuse; cognitive impairment; therapeutic community; residential treatment; cognitive screening; head injury 


\section{Introduction}

Therapeutic communities (TCs) are a form of residential rehabilitation for individuals experiencing chronic and severe alcohol and other drug (AOD) problems and may particularly suit clients with associated comorbidities and other complexities as they place an emphasis on fostering social support within the community of residents in order to promote treatment engagement and completion. Dropout from AOD treatment in general is estimated to be as high as $57 \%$ in inpatient settings (Darke, Campbell, \& Popple, 2012a; Deane, Wootton, Hsu, \& Kelly, 2012; Vergara-Moragues, Gonzalez-Saiz, Lozano, \& Verdejo Garcia, 2013). Importantly, length of stay in TCs has been shown to be related to neurocognitive capacity (Fals-Stewart \& Lucente, 1994; Fals-Stewart \& Schafer, 1992; Fernández-Serrano, Pérez-García, Perales, \& Verdejo-García, 2010a). An extensive systematic review reported that cognitive deficits were one of the most consistently reported risk factors for dropout from AOD treatment, alongside personality disorder, low treatment alliance, and younger age (Brorson, Ajo Arnevik, Rand-Hendriksen, \& Duckert, 2013).

It is likely that most residential substance misuse programs deliver treatments that work optimally in those who possess intact cognitive abilities; that cognitive impairment among residents may hinder treatment success requires further consideration. Components of residential AOD treatment often rely on capacities such as executive function (defined broadly as "those capacities that enable a person to engage successfully in independent, purposive, self-directed, and self-serving behavior”; Lezak, Howieson, Bigler, \& Tranel, 2012, p. 37) and working memory (Fernández-Serrano et al., 2010b; Yücel \& Lubman, 2007), as clients are required to integrate new information, formulate goals, establish new behavioural strategies, and plan for the future in overcoming addiction.

However, most misused substances impair attention, learning and memory, visuospatial abilities, and executive functioning, with perhaps the most robust deficits across 
all substances evident in inhibitory control, working memory, and decision-making (Fernández-Serrano, Pérez-García, Río-Valle, \& Verdejo-García, 2010b; Yücel \& Lubman, 2007; Yücel, Lubman, Solowij, \& Brewer, 2007). Structural and functional brain changes may occur after substance misuse (Broyd, van Hell, Yücel, \& Solowij, in press; Caplan, Epstein, Quinn, Stevens, \& Stern, 2007; Ersche \& Sahakian, 2007; Gonzalez, 2007; Gruber, Silveri, \& Yurgelun-Todd, 2007; Lorenzetti, Solowij, \& Yücel, in press; Oscar-Berman \& Marinkovic, 2007; Scott et al., 2007). Traumatic brain injury (TBI) is also highly prevalent in substance misuse populations, leading to significant complexities in the process of AOD treatment (Sacks et al., 2009; Solomon \& Malloy, 1992; Walker, Cole, Logan, \& Corrigan, 2007; West, 2011).

Although cognitive dysfunction is common in AOD samples and increasingly recognised by staff, there is often insufficient time or resources to implement detailed neuropsychological assessments. Cognitive assessment may be instrumental in facilitating the detection of AOD clients with clinically significant cognitive impairment, irrespective of aetiology. Assessing clients' cognitive capacities can inform subsequent implementation of strategies aimed at improving treatment retention and outcomes.

In the AOD treatment environment a brief but valid and reliable measure is required. This would provide an indication of potential cognitive dysfunction and alert staff to the possibility that further neuropsychological assessment and/or treatment modifications may be indicated. The Montreal Cognitive Assessment (MoCA; Nasreddine et al., 2005) comprises 12 items that tap five key neuropsychological domains: executive function, working memory, short-term memory, language, and visuospatial ability. The MoCA takes 15 minutes to administer, is a free resource (Nasreddine, 2014), and can be administered by staff without formal neuropsychological training. Initial studies have found evidence supporting the use of the MoCA in AOD treatment settings (Copersino et al., 2009; Copersino et al., 2012). 
The current study was exploratory in nature and sought to provide a naturalistic overview of a sample of Australian TC residents, with the MoCA administered as a brief screening measure to assess neuropsychological functioning. Performance on the MoCA was investigated in relation to demographic, substance use, psychological, and other variables related to impairments in cognition, including TBI.

\section{Material and methods}

\subsection{Participants}

The AOD group was recruited from We Help Ourselves (WHOs), a large provider of residential AOD treatment in Australia that uses the Therapeutic Community model of treatment. Participants were recruited across seven WHOs sites in New South Wales (Sydney, Hunter Valley) and Queensland (Sunshine Coast). The response rate was approximately $90 \%$. Specific inclusion and exclusion criteria were not applied in order to capture the heterogeneous and complex presentations of residential AOD treatment populations and to increase the generalisability of results. Although there was no formal assessment of substance use disorders using the Diagnostic and Statistical Manual of Mental Disorders (5th ed.; DSM-5; American Psychiatric Association, 2013) criteria it was assumed that residents would meet these criteria given their attendance at a high-intensity residential program.

A control group was recruited through University of Wollongong College, a provider of educational programs to persons who have not completed highschool matriculation, as an alternative pathway to tertiary study. All students were invited to participate and the response rate was approximately 60\%. Control participants, all native English speakers, were excluded for any lifetime dependence on or treatment for alcohol or other drugs, and any psychiatric or neurological diagnoses. 


\subsection{Procedure}

After a group information session, interested participants provided written consent and were individually assessed in a quiet testing room. This was conducted within the TC facility for the AOD group, and at a University psychology clinic for the control group. The MoCA was administered in approximately 15 minutes, adhering to the administration and scoring guidelines (Nasreddine, 2014). Additional information (e.g., basic demographics, history of head injury, current and past substance use for controls) was obtained through a ten-minute semi-structured interview and the Kessler Psychological Distress Scale (K10; Kessler et al., 2002) was administered, as well as the Severity of Dependence Scale (SDS; Gossop et al., 1995) for the AOD group. Control participants received a \$20 gift card as reimbursement for their time.

\subsection{Measures}

\subsubsection{MoCA}

The MoCA was used to assess neuropsychological functioning across its purported five domains (executive function, working memory, short-term memory, language and visuospatial ability). Executive function is assessed using trail-making, phonemic fluency, and verbal abstraction tasks. Working memory is assessed using sustained attention, serial subtraction, and digit span forwards/backwards tasks. Short-term memory is assessed through the delayed recall of five nouns. Language is assessed using naming (low familiarity animals), sentence repetition, and the phonemic fluency task. Visuospatial ability is assessed using clock-drawing and cube-copying tasks. The MoCA has displayed acceptable reliability in clinical groups (Bernstein, Lacritz, Barlow, Weiner, \& DeFina, 2011; Freitas, Simões, Marôco, Alves, \& Santana, 2012). Outcome measures included total MoCA score and domain subscores.

\subsubsection{Clinical variables}


The SDS (Gossop, Best, Marsden, \& Strang, 1997) measured AOD participants’ level of dependence on their primary substance of misuse. The K10 was used to capture level of psychological distress experienced by all participants. The K10 has been extensively used in both community and clinical samples as an indicator of a potential psychological disorder, and has demonstrated acceptable psychometric properties (George, Kinner, Bruno, Degenhardt, \& Dunn, 2010; Hides et al., 2007; Sunderland, Mahoney, \& Andrews, 2012). Relevant demographic and substance use data routinely collected by WHOs were accessed, following participants and the director of the service providing written consent.

\subsection{Data analysis}

All data were analysed using IBM Statistical Package for the Social Sciences (version 19; IBM Corp, 2010). The primary goals of the analysis were to compare the performance of residents and controls on the MoCA and to explore variation in MoCA scores and potential predictors (e.g., primary substance of misuse, gender effects) within the AOD sample.

Shapiro-Wilk statistics and visual inspection of histograms and boxplots determined that total MoCA score, MoCA domain subscores, age, years of education, K10 and SDS scores were not normally distributed for both AOD and control groups. Consequently, nonparametric techniques were employed for most of the analyses. Nonparametric analysis of covariance was required for some analyses to enable covarying demographic characteristics that differed between groups. Quade’s (1967) distribution-free procedure was used as a nonparametric alternative to analysis of covariance (see Olejnik \& Algina, 1985). This involved ranking the dependent variable and all covariates for all cases, ignoring the grouping variable. Following this, the linear regression of the ranked dependent variable on the ranked covariate measure was calculated and the unstandardised residuals saved, again ignoring the grouping factor. To calculate Quade's F statistic, a one-way analysis of variance using the 
residuals from the regression as the dependent variable and the grouping variable as the factor was performed.

Total MoCA score and cognitive domain subscores were the primary dependent variables for analysis. However, factor analyses of the MoCA have demonstrated that domains may be grouped differently (e.g., Duro, Simões, Ponciano, \& Santana, 2010; Freitas et al., 2012). Given the multi-process nature of neuropsychological tasks, whereby any single task may tap into a range of perceptual, cognitive and motor abilities, and the overlapping nature of the cognitive domains assessed by the MoCA (Freitas et al., 2012), we created an additional score for analysis of executive function. This was of particular interest because executive dysfunction is especially common in substance misusing individuals (e.g., Fernández-Serrano et al., 2010b; Gierski et al., 2013; Hester, Lubman, \& Yücel, 2010; Perry et al., 2011). As such, we utilised the standard measure of executive function from the MoCA as well as an extended measure that included performance on the visuospatial cube-copying and clock-drawing tasks, with the rationale that these tasks rely on key component processes of executive function.

All AOD residents were compared to controls in the first instance. Residents were then compared in terms of gender and primary substance of misuse. Primary substance of misuse groupings were formed by choosing the three largest representative groups in residence at the time of testing (alcohol, heroin and amphetamines). A broader opiates group was then formed by the addition of clients on methadone maintenance and buprenorphine users. Cocaine users, minimal in Australian AOD samples (Australian Institute of Health and Welfare, 2005), were grouped with amphetamine users to form a stimulants group. This strategy maximised group sizes for comparison, grouping together substances with similar chemical properties. However, primary users of cannabis, tranquilisers and benzodiazepines were not grouped or included in these comparisons due to insufficient sample sizes of these 
subgroups (see Results). Finally, AOD users hospitalised after a head injury were compared to those without serious head injuries.

\section{Results}

\subsection{Preliminary analyses}

MoCA data were available for 128 AOD residents and 37 controls. For some analyses, the AOD sample was reduced to 95 due to missing data (e.g. primary substance of misuse, n=16; SDS and K10 scores, n=33). Table 1 displays demographic, substance use, psychological, and cognitive functioning variables for the AOD group. AOD residents were in their mid-thirties on average and predominantly male. Alcohol was the primary substance of misuse for almost one-third of the sample, followed by heroin and amphetamine misuse, with these three substances accounting for approximately $80 \%$ of the entire AOD sample. The mean K10 score indicated very high levels of psychological distress and the mean SDS score indicated high levels of psychological dependence on participants’ primary substance of misuse, although the range extended to 0 in some cases, reflecting that some residents had progressed further in their treatment. The prevalence of head injuries was particularly high in the sample. Of the $67.2 \%$ who had either lost consciousness or sustained a concussion after a head injury, the mean number of times this had occurred was $4.98(\mathrm{SD}=5.95)$. Half of the total sample had been hospitalised after sustaining a head injury. 


\section{Table 1}

Demographic, substance use, psychological, and clinical variables for the AOD group $(N=128)$

\begin{tabular}{|c|c|}
\hline Age (Mdn, range) & $35(19-56)$ \\
\hline Gender (\% male) & 70.3 \\
\hline Education (Mdn, range) & $10(7-16)$ \\
\hline Unemployed (\%) & 90.5 \\
\hline Homeless $^{\mathrm{a}}(\%)$ & 7.4 \\
\hline Arrested during last three months ${ }^{\mathrm{a}}(\%)$ & 43.2 \\
\hline \multicolumn{2}{|l|}{ Primary substance of misuse ${ }^{b}(\%)$} \\
\hline Alcohol & 30.4 \\
\hline Heroin & 27.7 \\
\hline Amphetamines & 20.5 \\
\hline Cannabis & 8.9 \\
\hline Tranquilisers & 4.5 \\
\hline Methadone & 2.7 \\
\hline Buprenorphine & 1.8 \\
\hline Cocaine & 1.8 \\
\hline Benzodiazepines & 1.8 \\
\hline SDS $^{\mathrm{c}}$ score $^{\mathrm{a}}(\mathrm{Mdn}$, range $)$ & $11(0-15)$ \\
\hline History of overdose (\%) & 60.2 \\
\hline Injected during last three months (\%) & 52.6 \\
\hline $\mathrm{K}_{10}{ }^{\mathrm{d}}$ score $^{\mathrm{a}}(M, S D)$ & $29.2(7.8)$ \\
\hline Hospitalised after head injury (\%) & 50 \\
\hline Lost consciousness/concussion after head injury (\%) & 67.2 \\
\hline
\end{tabular}

All control participants were screened to ensure that they had not been dependent on alcohol or other substances and/or received treatment for substance use disorder in the past. No control participant currently used any substance (excluding alcohol and tobacco) on a regular basis (i.e., greater than once a month), with minimal prior experimentation with drugs, and $76 \%$ drank alcohol less than twice per week. The $24 \%$ of control participants who drank alcohol more than once per week were screened to ensure that their current drinking was not occurring at a problematic level that caused clinically significant impairment or distress. Despite attempts to match sample characteristics, controls were significantly 
younger $(\mathrm{Mdn}=25$ years, range 21-61) than the AOD group (Mdn = 35 years, range 19-56), $\mathrm{z}=-5.21, \mathrm{p}<.001$, and had significantly more years of education $(\mathrm{Mdn}=12$ years vs. $\mathrm{Mdn}=$ 10 years), $\mathrm{z}=-4.26, \mathrm{p}<.001$. Gender distribution did not differ significantly between groups (54\% male in controls vs. 70.3\% male in AOD). Age and education were therefore included as covariates for group comparisons. Psychological distress was low to moderate in controls (K10 mean 17), and was significantly higher in AOD residents (K10 mean 29), $\mathrm{F}(1,130)=$ 33.33, $\mathrm{p}=<.001$.

\subsection{Primary analyses}

\subsubsection{Covariates}

Despite the group difference in age, age was not significantly correlated with total MoCA score, $p=.16$. It was also not correlated with any MoCA subscores, all $p>.05$, in the entire sample as well as in the AOD group alone. There were significant correlations between years of education and total MoCA score, $r=.18, p=.02$, executive function, $r=.36, p<$ .001 , short-term memory, $r=.18, p=.02$, working memory, $r=.21, p=.006$, and language, $r=.26, p=.001$. Age and education were both included as covariates in analyses, but while education was consistently significant in the models, age was not. Age was subsequently dropped from the analyses and results are reported here with education alone as the covariate in analyses of MoCA outcomes for the AOD vs. control group.

\subsubsection{MoCA scores}

Comparison of the AOD and control group determined a significant difference in total MoCA score, $F(1,163)=5.28, p=.023$, the combined executive function/visuospatial subscore, $\mathrm{F}(1,163)=6.91, \mathrm{p}=.009$, and the visuospatial domain alone, $\mathrm{z}=-2.43, \mathrm{p}=.015$, with poorer performance in the AOD group (Table 2). None of the other cognitive domain subscores differed between groups: short-term memory, $F(1,163)=.25, \mathrm{p}=.62$, working 
memory, $\mathrm{F}(1,163)=.27, \mathrm{p}=.60$, and language, $\mathrm{F}(1,163)=1.04, \mathrm{p}=.31$. There was a trend toward poorer executive function in the AOD group, $\mathrm{F}(1,163)=3.01, \mathrm{p}=.08$.

Table 2

Comparison of MoCA scores between AOD and control groups, for entire AOD sample and those without head injuries: mean (SD)

\begin{tabular}{|c|c|c|c|}
\hline & $\underline{\text { Head Injuries included }}$ & Head injuries excluded & \\
\hline & AOD group $(N=128)$ & AOD group $(N=64)$ & Control $(N=37)$ \\
\hline Total score & 25.60 (3.13)* & $26.39(2.56)$ & $26.94(3.10)$ \\
\hline Executive/Visuospatial & $5.13(1.57)^{* *}$ & $5.53(1.47)$ & $6.14(1.64)$ \\
\hline Executive & $2.48(1.05)$ & $2.73(1.03)$ & $3.08(0.92)$ \\
\hline $\mathrm{STM}^{\mathrm{a}}$ & $3.63(1.33)$ & $3.78(1.23)$ & $3.92(1.23)$ \\
\hline$W^{\mathbf{b}}$ & $5.36(1.06)$ & $5.50(1.04)$ & $5.43(0.99)$ \\
\hline Visuospatial & $2.64(1.01)^{*}$ & $2.80(0.98)$ & 3.05 (1.13) \\
\hline Language & $5.01(0.94)$ & $5.16(0.88)$ & $5.35(0.82)$ \\
\hline
\end{tabular}

$* \mathrm{p}<.05 * * \mathrm{p}<.01$

a Short-term memory

${ }^{\mathbf{b}}$ Working memory

\subsubsection{Gender effects}

There were no significant differences between male and female AOD participants in age, years of education, or SDS scores (all $\mathrm{p}>.28$ ). Females tended to have higher K10 scores $(\mathrm{M}=31.25, \mathrm{SD}=7.89)$ than males $(\mathrm{M}=28.21, \mathrm{SD}=7.60), \mathrm{t}(93)=-1.82, \mathrm{p}=.07$. MoCA outcomes for males vs. females were not significantly different (all $p>.10$ ) although females tended to score slightly higher than males (Mdn 3 vs. 2) on executive subscores, $\mathrm{z}=$ $1.68, \mathrm{p}=.09$.

\subsubsection{Primary substance of misuse}

There were significant differences between primary substance of misuse groups in age, $\mathrm{F}(2,92)=8.25, \mathrm{p}=.001$, but not years of education, $\mathrm{H}(2)=4.15, \mathrm{p}=.13$, or SDS scores, $\mathrm{H}(2)=1.59, \mathrm{p}=.45$; age was therefore included as a covariate in the analysis. There were no significant differences in total MoCA score, $\mathrm{F}(2,92)=.04, \mathrm{p}=.96$, short-term memory, $\mathrm{F}(2$, $92)=.09, \mathrm{p}=.92$, language, $\mathrm{F}(2,92)=.15, \mathrm{p}=.86$, executive function, $\mathrm{F}(2,92)=1.37, \mathrm{p}=$ 
.26 , visuospatial abilities, $\mathrm{F}(2,92)=1.61, \mathrm{p}=.21$, or combined executive function/visuospatial abilities, $\mathrm{F}(2,92)=1.30, \mathrm{p}=.28$, between primary substance of misuse groups (Table 3). There was a marginally significant difference for working memory performance, $\mathrm{F}(2,92)=2.98, \mathrm{p}=.056$, with the poorest performance in the stimulants group. K10 scores were significantly different across substance of misuse groups, $F(2,77)=5.96$, $p$ $=.004$, being higher in the stimulant group relative to the opiate group $(\mathrm{p}=.022)$ and the alcohol group $(\mathrm{p}=.07)$, with no difference between alcohol and opiate groups $(\mathrm{p}=.69)$.

Table 3

Comparison of MoCA scores across primary substance of misuse groups: mean (SD)

\begin{tabular}{lccc} 
& Alcohol $(N=34)$ & Opiates $(N=36)$ & Stimulants $(N=25)$ \\
Total score & $25.85(3.49)$ & $25.92(2.55)$ & $25.88(3.23)$ \\
Executive/Visuospatial & $5.09(1.73)$ & $5.14(1.51)$ & $5.60(1.58)$ \\
Executive & $2.26(1.14)$ & $2.64(1.05)$ & $2.68(1.11)$ \\
$\mathrm{STM}^{\mathbf{a}}$ & $3.76(1.18)$ & $3.69(1.35)$ & $3.64(1.31)$ \\
$\mathrm{WM}^{\mathbf{b}}$ & $5.53(1.08)$ & $5.56(0.69)$ & $5.00(1.22)$ \\
Visuospatial & $2.82(0.94)$ & $2.50(1.06)$ & $2.92(0.91)$ \\
Language & $5.12(0.95)$ & $4.94(1.01)$ & $4.96(0.93)$ \\
\hline
\end{tabular}

\footnotetext{
a Short-term memory.

${ }^{\mathbf{b}}$ Working memory; trend toward greater impairment in the stimulants group $\mathrm{p}=.056$.
}

\subsection{Exploratory analyses}

\subsubsection{Psychological distress and substance dependence}

There were significant negative correlations between K10 scores and total MoCA scores, $r=-.22, p=.012$, executive function/visuospatial abilities, $r=-.21, p=.015$, and language scores, $r=-.20, p=.021$, and a trend for working memory, $r=-.16, p=.06$, in the overall AOD sample. K10 and SDS scores were positively correlated, $r=.20, p=.049$, but SDS scores were not correlated with any MoCA scores (all $p>.05$ ), other than a trend toward a correlation with executive function, $r=.18, p=.085$. This pattern indicates that greater psychological distress (but not greater drug dependence) is associated with greater cognitive impairment. 


\subsubsection{Head injuries}

Given that $50 \%$ of the AOD sample had been hospitalised for a head injury, the impact of head injuries on cognitive function were imperative to investigate further. There were no significant differences in age, $\mathrm{z}=-1.24, \mathrm{p}=.22$, education, $\mathrm{z}=-.94, \mathrm{p}=.35$, or $\mathrm{K} 10$ scores, $\mathrm{t}(93)=-.23, \mathrm{p}=.82$, between $\mathrm{AOD}$ residents who had versus had not been hospitalised after a head injury, but those who had not been hospitalised for head injury had higher SDS scores, $\mathrm{z}=-2.10, \mathrm{p}=.035,(\mathrm{Mdn}=11$ vs. 10$)$. AOD participants who had been hospitalised had lower total MoCA scores (Mdn $=25.5$ vs. 26), $\mathrm{z}=-2.59, \mathrm{p}=.01$, than those who had not. The hospitalised group also had lower executive/visuospatial (Mdn $=5$ vs. 6, $\mathrm{z}$ $=-2.97, \mathrm{p}=.003$ ), working memory (Mean Rank $=58.38$ vs. $70.62, \mathrm{z}=-2.18, \mathrm{p}=.03$ ), and executive ( $M d n=2$ vs. $3, z=-2.66, p=.008)$ subscores, than the non-hospitalised group, with trends toward lower language, $\mathrm{z}=-1.67, \mathrm{p}=.095$, and visuospatial subscores, $\mathrm{z}=-1.71$, $\mathrm{p}=.088$, but no difference in short-term memory subscores, $\mathrm{z}=-1.17, \mathrm{p}=.24$. Figure 1 displays standardised (out of 6) MoCA domain scores for AOD participants hospitalised for head injuries, non-hospitalised AOD participants and controls.

\subsubsection{Analysis of sample without significant head injuries}

After exclusion of those who had been hospitalised after a head injury, the primary analyses were repeated to compare only those AOD residents who had not sustained head injures requiring hospitalisation with controls (Table 2). The AOD group in this reduced sample $(n=64)$ were significantly older than controls (Mdn = 34 vs. 25 , respectively), $\mathrm{z}=$ 4.82, $\mathrm{p}=<.001$, and less educated ( $\mathrm{Mdn}=10$ vs. 12$), \mathrm{z}=-3.65, \mathrm{p}=<.001$. With age and education included as covariates, there were no significant differences in total MoCA score, $\mathrm{F}(1,99)=.38, \mathrm{p}=.54$, or any MoCA subscores (all $\mathrm{p}>.20$ ) between AOD residents without significant head injuries and controls. Significant differences remained for K10 scores between these AOD residents $(\mathrm{Mdn}=29)$ and controls $(\mathrm{Mdn}=16), \mathrm{F}(1,82)=33.51, \mathrm{p}=<$ 
.001. Other than males scoring significantly higher (Mean Rank $=36.43)$ in working memory subscores than females (Mean Rank $=25.50), \mathrm{z}=-2.91, \mathrm{p}=.004$, no other effects of gender or primary substance of misuse were found in this sample without head injuries.

\subsubsection{Clinical significance of impairment}

To investigate the clinical significance of impairment, total MoCA scores were recoded as a dichotomous variable to indicate either the presence or absence of cognitive impairment according to the cut-off score suggested by Copersino et al. (2009) as $\geq 26$ reflecting no evidence of cognitive impairment. Within the overall AOD group, 43.8\% met criteria for cognitive impairment. After removing those hospitalised for head injuries, 37.5\% met criteria for impairment and this represented a significantly greater proportion than the $16.2 \%$ of controls meeting criteria for impairment, $\chi^{2}(1, \mathrm{~N}=101)=5.09, \mathrm{p}=.019$. However, after removing a further 27 participants in the AOD sample who had sustained concussion or lost consciousness after a head injury but had not been hospitalised, the prevalence of impairment dropped to $29.7 \%$ and this did not represent a statistically significant difference when compared to the $16.2 \%$ of impaired controls $(p=.13)$. Subsequently, it was deemed appropriate to examine the prevalence of impairment within the AOD group who had been hospitalised after a head injury. Of those who had been hospitalised ( $n=64)$, only 50\% met criteria for cognitive impairment, indicating that hospitalisation for head injuries alone may not predict cognitive impairment. There was no difference in the total number of head injuries (including those that did not require hospitalisation) sustained by those who met criteria for impairment $(\mathrm{Mdn}=3)$ vs. those who did not $(\mathrm{Mdn}=3), \mathrm{p}=.67$.

\section{Discussion}

This study used a brief screening tool, the MoCA, to provide an overview of cognitive functioning in residents of a substance misuse TC. The major findings were that $43.8 \%$ of the 
AOD group met criteria for cognitive impairment and head injuries were a significant determinant of this impairment. Within the AOD group, 50\% had been hospitalised after a head injury and were more cognitively impaired than those who had not sustained head injuries requiring hospitalisation. Greater psychological distress in the AOD group was also associated with greater cognitive deficits.

\subsection{Neuropsychological functioning}

AOD residents overall scored significantly lower on the MoCA and showed greater deficits in executive function and visuospatial abilities than controls. No other cognitive domains differed between residents and controls. Within the AOD group, there were no gender effects other than a trend toward males displaying greater executive dysfunction than females and females tended to have higher levels of psychological distress than males. MoCA outcomes did not differ according to primary substance of misuse, other than stimulant users showing marginally poorer working memory ability and higher levels of psychological distress. The lack of observed differences in neuropsychological outcomes between substance of misuse groups may be explained by the prevalence of polysubstance use in clients undergoing residential AOD treatment; differences may not be detected between groups in which substance use has reached a level necessitating residential treatment. Generally, clients in residential AOD treatment have extensive histories of substance use with considerable variability between individuals. Another possible explanation is that differences do exist between substance of misuse groups in residential treatment but that the current study did not possess group sizes large enough to detect these differences.

\subsubsection{Head injuries}

Half of the AOD sample in the current study had been hospitalised after a head injury and were more cognitively impaired than those who had not, with lower total MoCA scores, and poorer executive/visuospatial and working memory abilities. When the hospitalised 
group were excluded from the analysis, there were no differences in any neuropsychological outcomes between AOD residents and controls, and no differences between primary substance of misuse groups, but females in this subset displayed poorer working memory performance than males.

In general, these findings are congruent with the literature suggesting that cognitive impairments exist in AOD populations (Caplan et al., 2007; Ersche \& Sahakian, 2007; Fernández-Serrano et al., 2010b; Gonzalez, 2007; Gruber et al., 2007; Oscar-Berman \& Marinković, 2007; Scott et al., 2007; Yücel \& Lubman, 2007; Yücel et al., 2007) and may be detected by a brief cognitive impairment-screening tool (the MoCA). In assessing a residential AOD sample, arguably we accessed those most severely affected by their addiction, and also those with a high prevalence of head injuries requiring hospitalisation. The level of cognitive impairment detected in this sample, as measured by the MoCA, was not severe and was largely associated with TBI, a factor that has not routinely been considered in previous studies and may account for a significant portion of the deficits observed in the literature.

\subsection{Implications}

This study showed that having sustained a TBI requiring hospitalisation accounted for the majority of cognitive impairment detected in the sample. There are two major implications: first, simply asking clients whether they have ever sustained a head injury requiring hospitalisation may be the most time-efficient way to informally gauge the possibility of cognitive deficits. Clients may be unable, however, to provide accurate accounts due to the high rate of TBIs occurring during intoxication that may remain undetected and receive no medical intervention. Whilst half of the AOD group in the current study had sustained a head injury requiring hospitalisation, 50\% of this subgroup did not meet criteria for impairment based on the MoCA score threshold. As such, screening for head 
injuries alone without subsequent cognitive assessment may lead to inflated estimates of impairment. Furthermore, this type of screening would not capture approximately one third of the AOD sample that had never sustained head injuries and yet met criteria for cognitive impairment. Thus, the second implication is that cognitive deficits may also exist independently of head injuries in AOD samples and hence require screening in their own right.

These complex findings highlight the importance of brief cognitive screening as a standardised assessment procedure for all clients entering residential treatment. In this way, treatment planning may account for the presence of cognitive deficits. Further neuropsychological assessment may be recommended and modifications to treatment and/or targeted interventions may be implemented.

Regardless of the aetiology of cognitive impairment, cognitive screening has the potential to inform interventions aimed at alleviating these deficits. For example, adjunct interventions to remediate cognitive deficits together with better-tailored specific treatments may bolster residential services, reducing dropout rates and consequently improving treatment outcomes. Evidence suggests that the neuropsychological deficits associated with TBI can be alleviated through cognitive remediation interventions (Maas et al., 2013; Manley \& Maas, 2013; Tsaousides \& Gordon, 2009), and there is emerging evidence for the use of cognitive remediation programs in substance misuse populations as a way to improve treatment retention (Bates, Buckman, \& Nguyen, 2013; Fals-Stewart \& Lam, 2010; Rupp, Kemmler, Kurz, Hinterhuber, \& Fleischhacker, 2012; Verdejo-García, 2011; Wexler, 2011). Further research aimed at developing and trialling cognitive remediation programs for AOD populations is required and providing these interventions within residential treatment services would be most ideal (e.g., Gonçalves et al., 2014).

\subsection{Limitations and future directions}


There are several limitations of the current study. There were clear differences between the AOD group and controls (e.g., age, education, inferred socioeconomic status). While some of these differences were accounted for statistically, future research would benefit from the inclusion of better-matched control groups. The exploration of primary substance of misuse groups resulted in small sample sizes with reduced statistical power for comparison; larger samples in future studies may reveal substance-specific impairment. Another limitation of the current study is its failure to assess for psychiatric comorbidities, which are common in AOD populations and add substantial complexity to the process of treatment. The current study utilised the K10 as a measure of psychological distress and higher scores were associated with greater cognitive impairment. Future studies should include formal psychiatric diagnoses in order to examine their interaction with substance use disorders and head injuries in terms of cognitive outcomes. Additionally, the current study focused on hospitalisation and frequency of head injuries, but not their nature, severity or age of occurrence, which could be further examined in future studies. Also, despite the AOD sample being abstinent from AOD, there was no assessment of their duration of abstinence or length of stay in residential services prior to cognitive assessment; this, along with a more detailed substance use history (including age of onset and number of years of substance use) and objective measures of abstinence (e.g. urine testing in both AOD and control samples), could have provided further information regarding the nature and extent of the observed cognitive deficits. Finally, the potential lack of sensitivity of the MoCA to more specific cognitive deficits in AOD populations cannot be underestimated; the scores observed in this sample were not substantially below the cut-off indicative of impairment. Nevertheless, the MoCA can serve as a cost-effective screening tool that would detect severe deficits in those most requiring further neurocognitive assessment.

\subsection{Conclusion}


The current study identified that the MoCA may be usefully applied in the AOD treatment setting as a brief screening tool. We showed in a naturalistic snapshot of clients in AOD residential treatment that cognitive impairment is common and may be related to the effects of head injuries, which are also highly prevalent. Cognitive abilities such as executive function and working memory are important for AOD residents to engage meaningfully in treatment and achieve successful outcomes. If these capacities are compromised, residents may be more likely to drop out from treatment and fail to recover from their addiction. The first step in rectifying this situation is to improve the assessment and detection of those who present with cognitive deficits. This will help to inform modifications to treatment and/or cognitive remediation interventions that may be beneficial in accommodating and potentially remediating impairments in cognition, increasing the likelihood of treatment engagement and retention, and hopefully leading to long-term recovery from addiction. 


\section{Acknowledgements}

The authors would like to thank the staff and residents of We Help Ourselves. 


\section{References}

American Psychiatric Association. (2013). Diagnostic and statistical manual of mental disorders. (5th ed.). Washington, DC: Author.

Australian Institute of Health and Welfare. (2005). National drug strategy household survey: Detailed findings, cat. no. PHE 66. Canberra, Australia: Author.

Bates, M. E., Buckman, J. F., \& Nguyen, T. T. (2013). A role for cognitive rehabilitation in increasing the effectiveness of treatment for alcohol use disorders. Neuropsychol Rev., 23, 27-47.

Bernstein, I. H., Lacritz, L., Barlow, C. E., Weiner, M. F., \& DeFina, L. F. (2011). Psychometric evaluation of the Montreal Cognitive Assessment (MoCA) in three diverse samples. Clin Neuropsychol., 25, 119-126.

Brorson, H. H., Ajo Arnevik, E., Rand-Hendriksen, K., \& Duckert, F. (2013). Drop-out from addiction treatment: A systematic review of risk factors. Clin Psychol Rev., 33, 1010_ 1024.

Broyd, S., van Hell, H. H., Yücel, M., \& Solowij, N. In press. Acute and chronic effects of cannabinoids on cognition - a systematic review. Biol Psychiatry. (accepted 5 December 2015)

Caplan, J. P., Epstein, L. A., Quinn, D. K., Stevens, J. R., \& Stern, T. A. (2007). Neuropsychiatric effects of prescription drug abuse. Neuropsychol Rev., 17, 363-380.

Copersino, M. L., Fals-Stewart, W., Fitzmaurice, G., Schretlen, D. J., Sokoloff, J., \& Weiss, R. D. (2009). Rapid cognitive screening of patients with substance use disorders. Exp Clin Psychopharmacol., 17, 337-344.

Copersino, M. L., Schretlen, D. J., Fitzmaurice, G. M., Lukas, S. E., Faberman, J., Sokoloff, J., \& Weiss, R. D. (2012). Effects of cognitive impairment on substance 
abuse treatment attendance: Predictive validation of a brief cognitive screening measure. Am J Drug Alcohol Abuse., 38, 246-250.

Darke, S., Campbell, G., \& Popple, G. (2012a). Retention, early dropout and treatment completion among therapeutic community admissions. Drug Alcohol Rev., 31, 64-71.

Darke, S., Campbell, G., \& Popple, G. (2012b). Self-harm and attempted suicide among therapeutic community admissions. Drug Alcohol Rev., 31, 523-528.

Deane, F. P., Wootton, D. J., Hsu, C. \& Kelly, P. J. (2012). Predicting dropout in the first 3 months of 12-step residential drug and alcohol treatment in an Australian sample. J Stud Alcohol Drugs, 73, 216-225.

Duro, D., Simões, M. R., Ponciano, E., \& Santana, I. (2010). Validation studies of the Portuguese experimental version of the Montreal Cognitive Assessment (MoCA): Confirmatory factor analysis. J Neurol., 257, 728-734.

Ersche, K. D., \& Sahakian, B. J. (2007). The neuropsychology of amphetamine and opiate dependence: Implications for treatment. Neuropsychol Rev., 17, 317-336.

Fals-Stewart, W., \& Lam, W. K. K. (2010). Computer-assisted cognitive rehabilitation for the treatment of patients with substance use disorders: A randomized clinical trial. Exp Clin Psychopharmacol., 18, 87-98.

Fals-Stewart, W., \& Lucente, S. (1994). Effect of neurocognitive status and personality functioning on length of stay in residential substance abuse treatment: An integrative study. Psychol Addict Behav., 8, 179-190.

Fals-Stewart, W., \& Schafer, J. (1992). The relationship between length of stay in drug-free therapeutic communities and neurocognitive functioning. J Clin Psychol., 48, 539543. 
Fernández-Serrano, M. J., Pérez-García, M., Perales, J. C., \& Verdejo-García, A. (2010a). Prevalence of executive dysfunction in cocaine, heroin and alcohol users enrolled in therapeutic communities. Eur J Pharmacol., 626, 104-112.

Fernández-Serrano, M. J., Pérez-García, M., Río-Valle, J. S., \& Verdejo-García, A. (2010b). Neuropsychological consequences of alcohol and drug abuse on different components of executive functions. J Psychopharmacol., 24, 1317-1332.

Freitas, S., Simões, M. R., Marôco, J., Alves, L., \& Santana, I. (2012). Construct validity of the Montreal Cognitive Assessment (MoCA). J Int Neuropsychol Soc., 18, 242-250.

George, J., Kinner, S. A., Bruno, R., Degenhardt, L., \& Dunn, M. (2010). Contextualising psychological distress among regular ecstasy users: The importance of sociodemographic factors and patterns of drug use. Drug Alcohol Rev., 29, 243-249.

Gierski, F., Hubsch, B., Stefaniak, N., Benzerouk, F., Cuervo-Lombard, C., Bera-Potelle, C., Cohen, R., Kahn, J.-P., \& Limosin, F. (2013). Executive functions in adult offspring of alcohol-dependent probands: Toward a cognitive endophenotype? Alcohol Clin Exp Res., 37, E356-E363.

Gonçalves, P. D., Ometto, M., Bechara, A., Malbergier, A., Amaral, R., Nicastri, S., Martins, P. A. (2014). Motivational Interviewing combined with chess accelerates improvement in executive functions in cocaine dependent patients: A one-month prospective study. Drug Alcohol Depend., 14, 79-84.

Gonzalez, R. (2007). Acute and non-acute effects of cannabis on brain functioning and neuropsychological performance. Neuropsychol Rev., 17, 347-361.

Gossop, M., Best, D., Marsden, J., \& Strang, J. (1997). Test-retest reliability of the Severity of Dependence Scale. Addiction, 92, 353-354. 
Gossop, M., Darke, S., Griffiths, P., Hando, J., Powis, B., Hall, W., \& Strang, J. (1995). The Severity of Dependence Scale (SDS): Psychometric properties of the SDS in English and Australian samples of heroin, cocaine and amphetamine users. Addiction, 90, 607-614.

Gruber, S. A., Silveri, M. M., \& Yurgelun-Todd, D. A. (2007). Neuropsychological consequences of opiate use. Neuropsychol Rev., 17, 299-315.

Hester, R., Lubman, D. I., \& Yücel, M. (2010). The role of executive control in human drug addiction. Curr Top Behav Neurosci., 3, 301-318.

Hides, L., Lubman, D. I., Devlin, H., Cotton, S., Aitken, C., Gibbie, T., \& Hellard, M. (2007). Reliability and validity of the Kessler 10 and Patient Health Questionnaire among injecting drug users. Aust N Z J Psychiatry, 41, 166-168.

IBM Corp. (2010). IBM SPSS Statistics for Windows, version 19.0. Armonk, NY: Author.

Kessler, R. C., Andrews, G., Colpe, L. J., Hiripi, E., Mroczek, D. K., Normand, S. L. T., Walters, E. E., \& Zaslavsky, A. M. (2002). Short screening scales to monitor population prevalences and trends in non-specific psychological distress. Psychol Med., 32, 959-976.

Lezak, M. D., Howieson, D. B., Bigler, E. D., \& Tranel, D. (2012). Neuropsychological assessment. (5th ed.). New York, NY: Oxford University Press.

Lorenzetti, V., Solowij, N., \& Yücel, M. In press. The role of cannabinoids on brain structural alterations in cannabis users. Biol Psychiatry. (accepted 12 November 2015)

Maas, A. I. R., Murray, G. D., Roozenbeek, B., Lingsma, H. F., Butcher, I., McHugh, G. S., Weir, J., Lu, J., \& Steyerberg, E. W. (2013). Advancing care for traumatic brain injury: Findings from the IMPACT studies and perspectives on future research. Lancet Neurol., 12, 1200-1210. 
Manley, G. T., \& Maas, A. I. R (2013). Traumatic brain injury: An international knowledge-based approach. J Amer Med Assoc., 310, 473-474.

Nasreddine, Z. S. (2015). The Montreal Cognitive Assessment (MoCA). Retrieved from http://www.mocatest.org/ on 17 December 2015.

Nasreddine, Z. S., Phillips, N. A., Bédirian, V., Charbonneau, S., Whitehead, V., Collin, I., Cummings, J. L., \& Chertkow, H. (2005). The Montreal Cognitive Assessment, MoCA: A brief screening tool for mild cognitive impairment. J Am Geriatr Soc., 53, 695-699.

Olejnik, S. F., \& Algina, J. (1985). A review of nonparametric alternatives to analysis of covariance. Eval Rev., 9, 51-83.

Oscar-Berman, M., \& Marinković, K. (2007). Alcohol: Effects on neurobehavioral functions and the brain. Neuropsychol Rev., 17, 239-257.

Perry, J. L., Joseph, J. E., Jiang, Y., Zimmerman, R. S., Kelly, T. H., Darna, M., Huettl, P., Dwoskin, L. P., \& Bardo, M. T. (2011). Prefrontal cortex and drug abuse vulnerability: Translation to prevention and treatment interventions. Brain Res Rev., 65, 124-149.

Quade, D. (1967). Rank analysis of covariance. J Am Stat Assoc., 62, 1187-1200.

Rupp, C. I., Kemmler, G., Kurz, M., Hinterhuber, H., \& Fleischhacker, W. W. (2012). Cognitive remediation therapy during treatment for alcohol dependence. J Stud Alcohol Drugs, 73, 625-634.

Sacks, A. L., Fenske, C. L., Gordon, W. A., Hibbard, M. R., Perez, K., Brandau, S., Cantor, J., Ashman, T., \& Spielman, L. A. (2009). Co-morbidity of substance abuse and traumatic brain injury. J Dual Diagn., 5, 404-417. 
Scott, J. C., Woods, S. P., Matt, G. E., Meyer, R. A., Heaton, R. K., Atkinson, J. H., \& Grant, I. (2007). Neurocognitive effects of methamphetamine: A critical review and meta-analysis. Neuropsychol Rev., 17, 275-297.

Solomon, D., \& Malloy, P. (1992). Alcohol, head injury, and neuropsychological function. Neuropsychol Rev., 3, 249-280.

Sunderland, M., Mahoney, A., \& Andrews, G. (2012). Investigating the factor structure of the Kessler Psychological Distress Scale in community and clinical samples of the Australian population. J Psychopathol Behav Assess., 34, 253-259.

Tsaousides, T., \& Gordon, W. A. (2009). Cognitive rehabilitation following traumatic brain injury: Assessment to treatment. Mt Sinai J Med., 76, 173-181.

Verdejo-García, A., (2011). Novel therapies for cognitive dysfunction secondary to substance abuse: Brief screening, referral, and cognitive rehabilitation. Psychiatr Times, 28, 46-48.

Vergara-Moragues, E., Gonzalez-Saiz, F., Lozano, O. M., \& Verdejo Garcia, A. (2013). Psychiatric profile of three-month retention in cocaine-dependent patients treated in a therapeutic community. J Stud Alcohol Drugs, 74, 452-459.

Walker, R., Cole, J. E., Logan, T. K., \& Corrigan, J. D. (2007). Screening substance abuse treatment clients for traumatic brain injury: Prevalence and characteristics. $J$ Head Trauma Rehabil., 22, 360-367.

West, S. L. (2011). Substance use among persons with traumatic brain injury: A review. Neurorehabilitation, 29, 1-8.

Wexler, B. E. (2011). Computerized cognitive remediation treatment for substance abuse disorders. Biol Psychiatry, 69, 197-198. 
Yücel, M., \& Lubman, D. (2007). Neurocognitive and neuroimaging evidence of behavioural dysregulation in human drug addiction: Implications for diagnosis, treatment and prevention. Drug Alcohol Rev., 26, 33-39.

Yücel, M., Lubman, D. I., Solowij, N., \& Brewer, W. (2007). Understanding drug addiction: A neuropsychological perspective. Aust NZ J Psychiatry, 41, 957-969. 